\title{
Proceso para priorizar las líneas de investigación esencial de interés para el Ministerio de Salud de Chile
}

\author{
RODOLFO ARMAS M. ${ }^{1,2}$, ADRIÁN TORRES C. .,a, JORGE ARRIAGADA C..$^{1, \mathrm{~b}}$, \\ FERNANDO MUÑOZ P. ${ }^{1}$, RODRIGO SALINAS R. ${ }^{1}$, PEDRO CROCCO A. ${ }^{1}$
}

\section{Definition of priorities in health research for the Ministry of Health}

Health research oriented to solve the most relevant sanitary problems in Chile must be encouraged. In 2001, the National Health Research Fund (FONIS) was created by the National Research Council of the Ministry of Health and the National Scientific Research Commission, to stimulate relevant health research that contributes to develop health care policies. In 2008 an experts meeting proposed eighty research areas. These areas were grouped in twelve thematic containers. Each of these containers were classified as having maximal, intermediate or minimal priority. The seven most important containers were grouped in three areas. Among the latter, two were selected. One is evaluation of the Ministry programs and, within this area, with the following priorities in decreasing importance: primary prevention, health care priorities, and diseases included in the Explicit Guarantees plan. The second area corresponds to diseases with high prevalence, incidence, costs or impact, including the following priorities in diminishing importance: mental health, diseases of high prevalence and problems with social impact.

(Rev Med Chile 2010; 138: 401-405).

Key words: Health Care research; Management information systems; Primary prevention.

$\mathrm{H}$ a habido una creciente toma de conciencia de parte de las autoridades sanitarias de estimular la investigación esencial en salud, entendiendo por tal aquella cuyo objetivo es resolver los problemas locales más urgentes. Esta ha sido conocida también como "investigación por necesidad" o "investigación por pertinencia".

Dentro de este contexto, diversas instituciones a nivel mundial crearon programas u organizaciones destinadas al fomento de la investigación aplicada o esencial. Así, surgieron entidades tales como el Consejo Asesor de Investigación en Salud (CAIS) de la Organización Panamericana de la Salud establecido en $1962^{1}$, el Council on Health Research for Development (COHRED) ${ }^{2}$ que es una organización no gubernamental con sede en Ginebra que inició sus actividades en 1993 y la fundación internacional independiente Global Forum on Health Research ${ }^{3}$ puesta en marcha en Ginebra en 1998 con el objeto de contribuir a la corrección de la brecha 10/90 en investigación en salud. La brecha 10/90 se ha mantenido pese a haber sido denunciada en 1998 y se refiere a que a nivel mundial, sólo $10 \%$ de los fondos utilizados en investigación científica en salud se destinan al 90\% de las enfermedades más letales de la humanidad.

El año 2001 el Ministerio de Salud de Chile creó el Consejo Nacional de Investigación en Salud (CONIS) ${ }^{4}$ el que, conjuntamente con la Comisión Nacional de Investigación Científica y Tecnológica (CONICYT), crearon el Fondo Nacional de Investigación y Desarrollo en Salud (FONIS) $)^{5,6}$. CONIS tiene por objetivo "asesorar 
en forma permanente al ministro(a) de salud respecto a temas prioritarios que sean orientadores en materia de investigación científica y desarrollo tecnológico en salud" y FONIS, proporcionar recursos concursables para las investigaciones aplicadas, cuyos resultados contribuyan a mejorar o modificar, tanto las políticas públicas sanitarias, como su implementación.

Así, aunque la investigación no es una tarea prioritaria del Ministerio de Salud, ella no le es ajena por cuanto es necesaria para el desarrollo técnico y la incorporación de conocimientos y tecnologías al trabajo médico, especialmente aquellos orientados a enfrentar necesidades prioritarias.

No obstante lo anterior, el Ministerio no ha priorizado las áreas o temas en los que debiera desarrollar actividad investigativa para mejor orientar sus recursos, que obviamente son limitados. En el curso del año 2008, el Consejo Nacional de Investigación acordó identificar tales áreas o temas y para ello, consultó a diversas autoridades que se relacionan con investigación en salud. Se hizo evidente que ésta era una tarea difícil, pues surgieron ideas muy distintas respecto de las patologías o grupos de patologías que debía priorizarse. Se propuso por ejemplo, dar preferencia a los problemas sanitarios derivados de la iniquidad social y económica de la población, a las patologías propias que no iban a ser suficientemente desarrollados en otros lugares del mundo, a las afecciones de mayor prevalencia e incidencia, a los aspectos de gestión y financiamiento que aportaran mayor eficiencia y eficacia a la atención médica, a los problemas de salud que tienen un fuerte impacto social, etc. Ante la diversidad de criterios y opiniones se optó por consultar a un grupo de expertos de distintas áreas para que libremente y sin restricciones temáticas propusieran las prioridades. El presente documento informa del proceso seguido para este fin y su resultado.

\section{Material y Método}

En una primera etapa, en abril de 2008, se realizó un seminario al que se invitó a ochenta personas consideradas expertas en el tema y provenientes de distintos ámbitos: Administración central del Ministerio de Salud y organismos asociados o dependientes de él tales como Instituto de Salud Pública, FONIS y Superintendencia de Salud; universidades públicas y privadas; dirección de los cuatro principales fondos de investigación del país (Fondo Nacional de Desarrollo Científico y Tecnológico, FONDECYT; Fondo Nacional de Investigación y Desarrollo en Salud, FONIS; Fondo de Fomento al Desarrollo Científico y Tecnológico, FONDEF); Corporación de Fomento de la Producción; sociedades científicas médicas y empresas privadas vinculadas a la salud. Asistieron $30(24 \%)$ de las 80 personas invitadas pero que cubrían todo el espectro de instituciones señaladas.

En esa oportunidad se presentaron tres exposiciones que explicaron la necesidad de precisar las materias en las que sería conveniente que el Ministerio enfatizara las investigaciones en salud, luego se sostuvo un debate al respecto, más tarde se solicitó a cada asistente que señalara cuáles serían a su parecer los tres temas o líneas de investigación que debieran merecer la mayor atención del Ministerio y, por último, se realizó tres talleres temáticos para profundizar y complementar lo anterior. Se obtuvo ochenta proposiciones de investigación; algunas eran de temas muy específicos y otras líneas de investigación de mayor o menor amplitud.

En una segunda etapa, se agrupó las ochenta propuestas en doce líneas amplias de investigación o contenedores temáticos (Tabla 1) y se practicó

\section{Tabla 1. Líneas de Investigación Propuestas}

1 Gestión y evaluación de programas

2 Patologías de alta prevalencia, incidencia y/o costos

3 Salud Mental

4 Problemas de salud del Adulto mayor

5 Salud del niño y el adolescente

6 Temas de salud que tienen importante impacto social

7 Inequidades en Salud

8 Desarrollo de la biotecnología

9 Desarrollo de la Investigación, formación de investigadores e institucionalización de la investigación

10 Estrategias empleadas en prevención primaria

11 Enfermedades zoonóticas

12 Grado de satisfacción laboral del personal de la salud y de la población en general respecto de su atención de salud 
electrónicamente un cuestionario tipo Likert ${ }^{7}$ a las treinta personas que participaron en la primera etapa, solicitándoles que señalaran para cada una de esas doce propuestas si merecía una prioridad máxima, una mínima o una intermedia para un apoyo ministerial. Se recibió 16 (53,3\%) respuestas.

En una tercera etapa se conformó tres grandes áreas o líneas de investigación que abarcaban los siete contenedores temáticos que tuvieron mayor preferencia en la encuesta y excluían a las 5 con menor preferencia. En una sesión a la que asistieron $16(100 \%)$ de las personas que habían respondido el cuestionario Likert, luego de un debate, se eligió a las dos que tenían la mayor preferencia. La elección se realizó mediante una votación en que cada asistente asignó prioridad 1, 2 ó 3 a cada una de las tres proposiciones.

El método aplicado en el proceso de consulta fue dirigido por uno de los autores (ATC).

\section{Resultados}

Las propuestas obtenidas en la primera etapa fueron 80 e incluían temas específicos y líneas de investigación de diferente amplitud.

En la segunda etapa, esas 80 propuestas se agruparon en 12 líneas de investigación y a través de un cuestionario de escalamiento Likert se obtuvo las preferencias ordenadas de mayor a menor indicadas en la Tabla 1.
Para una tercera etapa se reagruparon las siete líneas de investigación que resultaron con mayor prioridad, en tres grandes áreas que se indican en la Tabla 2 y que son: 1) Gestión y evaluación de programas; 2) Patologías de alta prevalencia, incidencia, costos y/o impacto social; 3) Patologías relacionadas con iniquidades sociales. Cada una de estas áreas tenía los contenidos que se señala en la Tabla 2. Como se aprecia, se excluyó las investigaciones relacionadas con enfermedades zoonóticas y las con indagación del grado de satisfacción laboral del personal de la salud y de la población en general respecto de su atención de salud, por no haber sido preferidas en la consulta realizada a través del cuestionario Likert.

En la reunión final, con asistencia de 16 expertos, luego de analizar el procedimiento seguido y sus resultados hasta esa reunión, los asistentes se pronunciaron sobre si daban primera, segunda o tercera prioridad a cada una de estas tres grandes áreas y se ponderó cada una de las opiniones. La opinión de los expertos apuntó mayoritariamente y con igual fuerza a priorizar las investigaciones relacionadas con gestión y evaluación de programas y las referentes a patologías de alta prevalencia, incidencia, costos y/o impacto social. Consultados sobre la mayor o menor prioridad de los contenidos de estas dos áreas, el orden de las tres mayores preferencias para las investigaciones relacionadas con gestión y evaluación de programas fue: a) prevención primaria; b) establecer prioridades en salud y c) patología incluida en el programa de

Tabla 2. Tres grandes áreas de investigación que incluyen a los temas que tuvieron mayor preferencia en la encuesta

\begin{tabular}{|ll|}
\hline Línea de Investigación & Contenido de la Línea de Investigación \\
$\begin{array}{ll}\text { 1. Gestión y evaluación de programas } & \text { a. Prevención primaria } \\
& \text { b. Establecer prioridades en salud } \\
\text { c. Patología incluida en el programa de Garantías Explícitas en Salud } & \text { d. Gestión y financiamiento de atención de salud y } \\
\text { e. Participación ciudadana en proceso de atención médica }\end{array}$ \\
$\begin{array}{ll}\text { 2. Patologías de alta prevalencia, } & \text { a. Asuntos relacionados con salud mental } \\
\text { incidencia, costos y/o impacto social } & \text { b. Patología pediátrica, de la adolescencia y del adulto mayor y } \\
\text { 3. Patologías relacionadas con } & \text { c. Problemas de salud con gran impacto social } \\
\text { inequidades sociales } & \text { Diversas condiciones sanitarias según características sociales, económicas, de } \\
& \text { género, de ruralidad o urbanismo de las personas, Ejemplos: } \\
\text { a. Desarrollo del niño } & \text { b. Acceso a la salud } \\
\text { c. Problemas de salud mental } \\
\text { d. Morbilidad y mortalidad general }\end{array}$ \\
\hline
\end{tabular}


Garantías Explícitas en Salud (AUGE/GES). Por su parte, para las patologías de alta prevalencia, incidencia, costos y/o impacto social, el orden de priorización de mayor a menor fue: a) patologías relacionadas con salud mental (droga adicción y alcoholismo, depresión y suicidio, delincuencia, conducta sexual y reproductiva, violencia intrafamiliar); b) afecciones de alta prevalencia sea pediátrica, de la adolescencia y del adulto mayor (hipertensión, diabetes, obesidad) y c) patologías de interés por su impacto social como VIH, desnutrición, tuberculosis.

\section{Discusión}

El procedimiento seguido fue una forma de consulta amplia a personas expertas y a quienes debiera interesar el tema.

Es probablemente inevitable que la selección de personas invitadas en este proceso y más aun las que se interesaron en participar en él, representen un sesgo con influencia en los resultados. En un estudio como éste, las personas consultadas deben conformar un grupo que represente equilibradamente a los determinantes sociales, económicos, culturales, demográficos y de la más diversa índole que interactúan en salud.

Es interesante que habiendo sido convocadas para participar en este proceso 80 personas, sólo 16 (20\%) de ellas lo hicieron en las tres etapas que éste tuvo. Probablemente quienes se desempeñan en la administración de salud no privilegian esfuerzos relacionados con investigación y quienes lo hacen en el mundo académico no se interesan mayormente en la investigación aplicada que realice el Ministerio de Salud. En todo caso, si bien hubo deserción de un número importante de personas invitadas, cualitativamente el grupo que cumplió las tres etapas tuvo representatividad de todos los sectores invitados ${ }^{8}$.

Cabe mencionar lo expresado respecto de salud mental en las dos reuniones de trabajo sostenidas en este proceso. En efecto, se señaló que un estudio de la Organización Mundial de la Salud situó a la ciudad de Santiago como una de las con mayor prevalencia de patología mental del mundo ${ }^{9}$, que las licencias médicas por causa psiquiátrica fueron la principal causa de ausentismo laboral en Chile durante 2007 y que uno de cada 3 ó 4 chilenos tendría un trastorno psiquiátrico en el curso de su vida ${ }^{10}$; contrastando con lo anterior, en el país las investigaciones relacionadas con salud mental son escasas y menos aun son las verdaderamente relevantes. Además, se comentó que dentro de salud mental se incluyen temas tan variados como psicopatías, depresión y suicidio, alcoholismo, droga adicción y quizás comportamientos sociales como son la delincuencia y enfrentamiento de la sexualidad entre los jóvenes. Se planteó que este tema podría quizás merecer un enfrentamiento especial como se hizo hace algunas décadas con nutrición infantil. Incluso se sugirió crear un Centro Nacional para investigación en Salud Mental.

De acuerdo a los resultados obtenidos en este trabajo, el Ministerio de Salud podría privilegiar las siguientes dos líneas de investigación: las relacionadas con gestión y evaluación de sus programas, especialmente las relacionadas con prevención primaria, prioridades en salud y patología incluida en el programa de Garantías Explícitas en salud y las referentes a patologías de alta prevalencia, incidencia, costos y/o impacto social, especialmente patologías relacionadas con salud mental (droga adicción y alcoholismo, depresión y suicidio, delincuencia, conducta sexual y reproductiva, violencia intrafamiliar) y afecciones de alta prevalencia como hipertensión, diabetes y obesidad.

Se comprende que las categorías establecidas en esta ordenación de temas no son excluyentes y que es muy posible que una determinada investigación pueda ser considerada en varias de ellas. Por ejemplo, un estudio referente a prevención de hipertensión arterial puede merecer ser prioritario por tratarse de prevención primaria, por ser patología incluida en el programa de Garantías Explícitas en Salud y por corresponder a una patología de alta prevalencia.

Las formas de privilegiar esos temas pueden ser diversas: orientar parte de los fondos del Fondo Nacional de Investigación en Salud hacia ellos, asignar un puntaje de premio en los concursos de ese fondo a los proyectos que se refieren a estos temas, procurar convenios con instituciones nacionales o extranjeras para que desarrollen investigaciones en estas materias, representar a las universidades la conveniencia de privilegiar estos temas para el desarrollo de tesis de postgrado, etc.

Esta es la primera vez que el Ministerio de Salud hace un intento por establecer sus propias prioridades en investigación. Es recomendable 
que un estudio de esta naturaleza se realice periódicamente, perfeccionando al actual en base a lo aprendido en esta oportunidad.

\section{Referencias}

1. http://www.paho.org/cais, Enero 2010.

2. http://www.cohred.ch, Enero 2010.

3. http://www.globalforumhealthresearch.org

4. Ministerio de Salud, Decreto No 145 de 2001.

5. Valdivieso V. El futuro de la investigación en Salud. Visión de las universidades. Boletín Academia Chilena de Medicina 2001; 38: 89-93.

6. Armas MR. Hacia un fondo Nacional de Investigaciones aplicadas en Salud, Boletín Academia Chilena de Medicina 2001; 38: 95-9.
7. Hernández $\mathrm{R}$, et al. Metodología de la Investigación. $2^{\mathrm{da}}$ Edic. Mc Graw-Hill, México 1991; pág 256 y ss.

8. Acuña H, Báez R, Konow I, Pérez G, Toloza C, Vera I. Métodos y Técnicas de investigación prospectiva para la toma de decisiones. Santiago, Chile: FUNTUROODEPLAN-PNUD. Enero de 1990

9. Fathalla M, Fathalla M. Guía práctica de investigación en Salud. Washington DC: Organización Panamericana de la Salud. 2008.

10. Hernández Sampieri R, Fernández-Collado C, Baptista Lucio P. Metodología de la Investigación (Cuarta Edición ed.). México, D.F., México: McGraw-Hill. 2008.

11. Goldberg DP, Lecrubier JF. Gors and frequency of mental disorders. 1995.

12. Vicente B, Rioseco P, Saldivia S, Kohn R, Torres S. Estudio chileno de prevalencia de patología psiquiátrica. Rev Med Chile 2002; 130: 527-36. 